\title{
microRNA-29a suppresses cell proliferation by targeting SPARC in hepatocellular carcinoma
}

\author{
XU-CHAO ZHU ${ }^{1,2}$, QIONG-ZHU DONG ${ }^{2}$, XIAO-FEI ZHANG ${ }^{2}$, BIAO DENG $^{2}$, \\ HU-LIANG JIA ${ }^{2}$, QIN-HAI YE ${ }^{2}$, LUN-XIU QIN $^{2}$ and XING-ZHONG WU ${ }^{1}$ \\ ${ }^{1}$ Department of Biochemistry and Molecular Biology, Shanghai Medical College, Fudan University, \\ Key Laboratory of Glycoconjugate Research, Ministry of Public Health, Shanghai 200032; \\ ${ }^{2}$ Liver Cancer Institute and Zhongshan Hospital, Institutes of Biomedical Sciences, Fudan University, \\ Key Laboratory of Carcinogenesis and Cancer Invasion, Ministry of Education, Shanghai 200032, P.R. China
}

Received June 1, 2012; Accepted July 25, 2012

DOI: $10.3892 /$ ijmm.2012.1140

\begin{abstract}
In the present study, we constructed a lentivirus vector encoding the miR-29a precursor and established two stably infected cell lines, PLC-29a and 97L-29a. The overexpression of miR-29a was confirmed by TaqMan RT-PCR and significantly suppressed the growth of the hepatocellular carcinoma cell lines MHCC-97L and PLC. Dual-luciferase reporter assays indicated that the SPARC mRNA 3'UTR was directly targeted by miR-29a since the mutated 3'UTR was not affected. Silencing SPARC expression by RNAi knockdown resulted in a similar effect as miR-29a overexpression on hepatocellular carcinoma (HCC) cell growth regulation. Anti-miR-29a oligonucleotides (AMOs) upregulated the levels of SPARC in the HCC cells. The phosphorylation of AKT/mTOR downstream of SPARC was inhibited in miR-29a-overexpressing HCC cells. We further examined and compared the expression levels of miR-29a in HCC tissues and the corresponding nearby noncancerous liver tissues of 110 patients with HCC by qRT-PCR, and significantly lower expression of miR-29a was observed in the tissues affected by HCC. Our findings demonstrate that the expression of miR-29a is important in the regulation of the SPARC-AKT pathway and HCC growth.
\end{abstract}

\section{Introduction}

Hepatocellular carcinoma (HCC) is one of the most common malignant tumors worldwide, particularly in Asian countries due to the prevalence of hepatitis B infection. Despite under-

Correspondence to: Professor Xing-Zhong Wu, Department of Biochemistry and Molecular Biology, Shanghai Medical College, Fudan University, 138 Yi Xue Yuan Road, Shanghai 200032, P.R. China E-mail: xz_wu@shmu.edu.cn

Dr Lun-Xiu Qin, Liver Cancer Institute and Zhongshan Hospital, Fudan University, 180 Feng Lin Road, Shanghai 200032, P.R. China E-mail: qin.lunxiu@zs-hospital.sh.cn

Key words: hepatocellular carcinoma, SPARC, miR-29a, AKT going multimodal treatment including partial hepatectomy, thermal ablation, radiation, chemotherapy, systemic chemotherapy and liver transplantation $(1,2)$, most of these patients eventually die from progressive tumors. Thus, an understanding of the molecular mechanisms involved in the tumor progression, development and growth regulation of HCC is urgently needed.

SPARC, also named osteonectin, is a secreted multifunctional matricellular glycoprotein and rich in cysteine. It is involved in important biological functions, including cell adhesion, migration, tissue repair and remodeling. SPARC is differentially expressed in varous tumors including breast cancer (3), melanoma (4), gliomas (5), prostate cancer (6) and colorectal cancer (7). However, the significance remains unclear and the detailed functions and molecular mechanisms are not known. Lau et al (8) observed that SPARC is related to HCC angiogenesis and tumor progression. Exogenous SPARC increases cell survival under stress initiated by serum withdrawal through a decrease in apoptosis and rapidly induces AKT phosphorylation, an effect that is blocked by a neutralizing SPARC antibody (5). Cell growth and proliferation are associated with AKT phosphorylation and activation. Furthermore, AKT activation is also essential for anti-apoptotic effects since AKT is involved in the regulation of tumor cell growth (9).

microRNAs (miRs) are small, non-coding RNA molecules that regulate gene expression (10), by translational inhibition or cleavage of their target mRNAs through base-pairing to partially or fully complementary seed sites. Aberrant miRNA expression, including expression of miR-21, miR-122a, miR-148, miR-185, miR-199a and miR-151, has been observed in HCCs and has been shown to regulate cell growth, apoptosis, migration, or invasion in different study cohorts (11-15). miR-29a is involved in renal fibrosis by suppressing collagen expression (16). miR-29a also appears to hinder elastin expression in fibroblasts and smooth muscle cells (17), which prevents and slows the development of fibrosis. miR-29a suppresses interferon- $\alpha$ receptor and regulates the threshold for infectionassociated thymic involution (18). In acute myeloid leukemia cells, upregulation of the nuclear oncogene Ski is associated with low miR-29a levels because forced expression of miR-29a downregulates Ski (19). Given that miR-29a is 
involved in myogenesis, osteoblastic differentiation, sclerosis and cardiac fibrosis $(14,19-22)$, there is also evidence showing that miR-29a is significantly downregulated in patients with HCC (14). Downregulation was mainly observed in the serum of fibrosis patients, targeting the pro-apoptotic factors Bcl-2 and Mcl-1. However, the detailed regulatory role of miR-29a in HCC tumorigenesis and metastasis is not fully understood and requires further investigation.

In this present study, we found that miR-29a expression was dramatically decreased in the majority of examined HCC tissues, and overexpression of miR-29a dramatically induced cell growth inhibition, which was associated with SPARC signaling in the AKT pathway.

\section{Materials and methods}

Cell lines and tissue specimens. Human HCC cell lines (PLC, MHCC-97H, MHCC-97L and SMMC-7721), the normal human hepatic cell line LO2, and HEK-293T cells were all cultured in Dulbecco's modified Eagle's medium (DMEM; Hyclone, Logan, UT, USA) supplemented with $10 \%$ fetal bovine serum (FBS; Gibco, Austria) in a humidified $37^{\circ} \mathrm{C}$ incubator with $5 \%$ $\mathrm{CO}_{2}$. A total of 110 patients with newly diagnosed $\mathrm{HCC}$ joined this study at the Liver Cancer Institute and Zhongshan Hospital, Fudan University (Shanghai, China) between 2003 and 2006. Samples of HCC and corresponding adjacent non-tumor liver tissues were collected after resection of the HCC. All of the clinical samples were collected from patients after obtaining informed consent according to an established protocol approved by the Ethics Committee of Fudan University.

Plasmids, lentivirus production and transduction. To construct a plasmid expressing miR-29a, the pri-miR-29a sequence was amplified with the primers AAAGGATCCGCCATAGAAACC CAGTTTC and AAAACGGCTCCAAGGGATGAATGTAA TTG, from human genomic DNA and then subcloned into the BamHI and MluI sites of the pWPI 1.1 vector to generate pWPImiR-29a. The wild-type SPARC-3'UTR sequence that contained putative binding site for miR-29a was PCR-amplified from oligo(dT)-primed HEK 293T cDNA with the primers ccgCTCGAGATCCACTCCTTCCACAGTACCG and ttGCGGCCGCGTGTGGTCTGCCTGCTAGA, and the 2.4-kb product was then subcloned into the XhoI and NotI sites of the psiCHECK-2 vector, immediately downstream of the Renilla luciferase gene. The resulting plasmid was named psi-SPARC3'UTR-WT. The plasmid psi-SPARC-3'UTR-MUT encodes a mutated sequence in the complementary site for the seed region of miR-29a, which was generated via site-directed mutagenesis (QuickChange XL site-directed mutagenesis kit; Stratagene, Cedar Creek, TX, USA) and psi-SPARC-3'UTR-WT as a template. The SPARC mutant site position was as follows: the first site position UCUGCCUGGAGACAAGGUGCUAA became UCUGCCUGGAGACAACCCCGCAA. The second site position AGUGAAUACAUUAACGGUGCUAA became AGUGAAUACAUUAACCCCCGCAA. Virus particles were harvested $60 \mathrm{~h}$ after pWPI-miR-29a transfection of the packaging plasmid psPAX2 and pMD2.G into HEK-293T cells using the FuGENE 6 reagent (Roche Diagnostics). PLC and MHCC-97L cells were infected with recombinant lentivirustransducing units and $8 \mu \mathrm{g} / \mathrm{ml}$ Polybrene (Sigma).
RNA extraction and quantitative RT-PCR. Total RNA was extracted from frozen tumor specimens and cell lines using TRIzol reagent (Invitrogen, Carlsbad, CA, USA) according to the manufacturer's instructions. Total RNA (500 ng) was reverse-transcribed using PrimeScript ${ }^{\circledR}$ RT Master Mix (Takara) reverse transcriptase according to the manufacturer's instructions. Real-time PCR analyses were performed with FastStart Universal SYBR-Green Master (Roche Diagnostics). For qRT-PCR of miR-29a, 50 ng of total RNA was reversetranscribed with miR-29a (ABI ID, 000412)- or U6 (ABI ID, 1973)-specific stem-loop primers and subjected to TaqMan miRNA assays using an ABI PRISM ${ }^{\circledR} 7900$ HT sequence detection system (from Applied Biosystems, Foster City, CA, USA).

Luciferase reporter assays. HEK293T cells plated in a 96-well plate were co-transfected with $200 \mathrm{ng}$ of pWPI.1 or pWPI-miR-29a and $40 \mathrm{ng}$ of psi-SPARC-3'UTR-WT or psi-SPARC-3'UTR-MUT. For the antagonism experiment, HEK293T cells were cultured in 96-well plates and co-transfected with $200 \mathrm{nM}$ anti-miR-C (control) or anti-miR-29a, as well as $40 \mathrm{ng}$ of psi-SPARC-3'UTR-WT or psi-SPARC-3'UTRMUT. Luciferase activity was measured $48 \mathrm{~h}$ after transfection and analyzed using the Dual-Luciferase reporter assay system (Promega). Transfections were performed in duplicate and repeated in at least three independent experiments.

Cell proliferation assays. Cell proliferation was determined using the WST-1 (Roche Diagnostics) assay. Cells (3x10³/well) were plated into the wells of a 96-well plate, each containing $90 \mu 1$ of culture medium. After 24, 48, 72 and $96 \mathrm{~h}, 10 \mu 1$ WST-1 was added, the cells were incubated for $2 \mathrm{~h}$ at $37^{\circ} \mathrm{C}$, and then the absorbance was measured at a wavelength of $450 \mathrm{~nm}$. Three independent experiments were performed.

Oligonucleotide (AMO) transfection. The small interfering RNA sequence targeting human SPARC was AUUGCUGCA CACCUUCUCA. The unspecific control siRNA (NC) was not homologous to any human genome sequences and synthesized by GenePharma Co., Ltd. (Shanghai, China). The anti-miR-29a was a 2'O-methyl-modified oligonucleotide with a sequence that was complementary to the mature miR-29a and was designed as an inhibitor of miR-29a. The anti-miR-C was used as a negative control in the antagonism experiments. Transfection of plasmid DNA or co-transfection of RNA duplexes with plasmid DNA was performed using the FuGENE 6 reagent. All transfections were performed in triplicate.

Western blotting. Cells were harvested and lysed with RIPA lysis buffer supplemented with protease inhibitors and phosphatase inhibitors (Roche Diagnostics). Protein extracts were separated by $10 \%$ SDS-PAGE and transferred to a polyvinylidene fluoride membrane (Millipore). The membrane was blocked with 5\% non-fat milk and incubated with rabbit anti-SPARC polyclonal antibody, rabbit anti-p-AKT (S473) p-mTOR (S2448) or P-ERK (T202, Y204) polyclonal antibodies, rabbit anti-AKT polyclonal antibody, or GAPDH rabbit antibody, all of which were from Cell Signaling Technology, Inc. The protein bands were detected with Chemiluminescent HRP substrate (Millipore). 

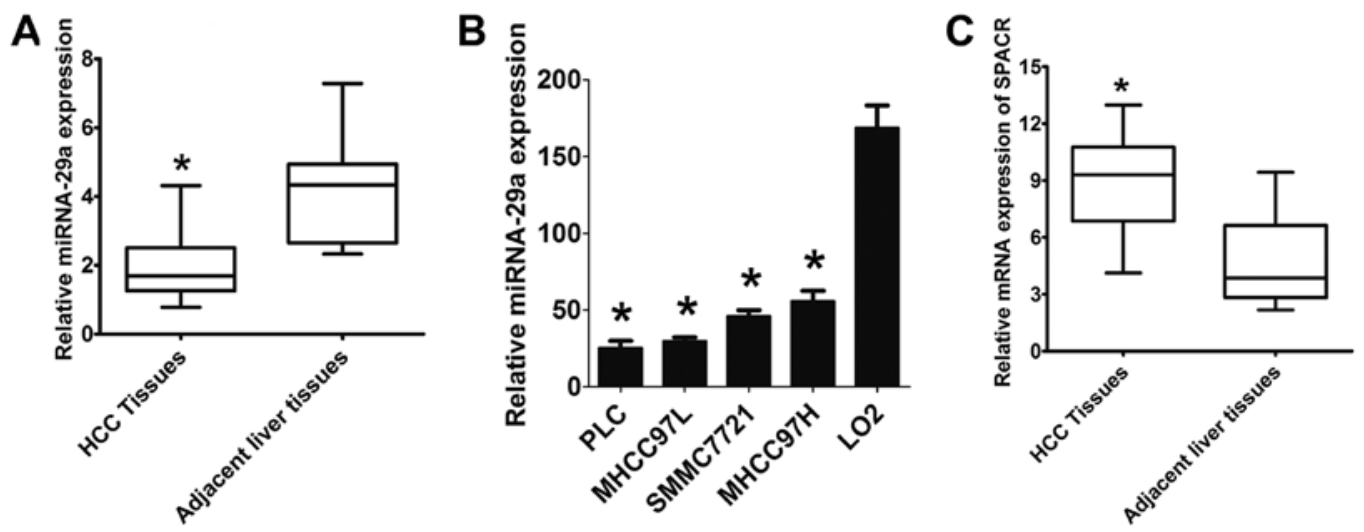

Figure 1. Expression analysis of miR-29a in HCC. (A) microRNA expression was determined by TaqMan qRT-PCR in HCC tissue and adjacent noncancerous liver tissue samples $(\mathrm{n}=110)$. Box plots describe the relative expression of miRNAs. (B) The expression levels of miR-29a in $4 \mathrm{HCC}$ cell lines and the normal liver cell line LO2. miR-29a expression was determined by TaqMan qRT-PCR. The miR-29a expression level was normalized to U6. (C) SPARC expression was determined by TaqMan qRT-PCR in HCC tissue and adjacent noncancerous liver tissue samples $(\mathrm{n}=110)$. Box plots describe the relative expression of the mRNA. Statistical analysis was performed using the paired Student's t-test. ${ }^{*} \mathrm{P}<0.05$
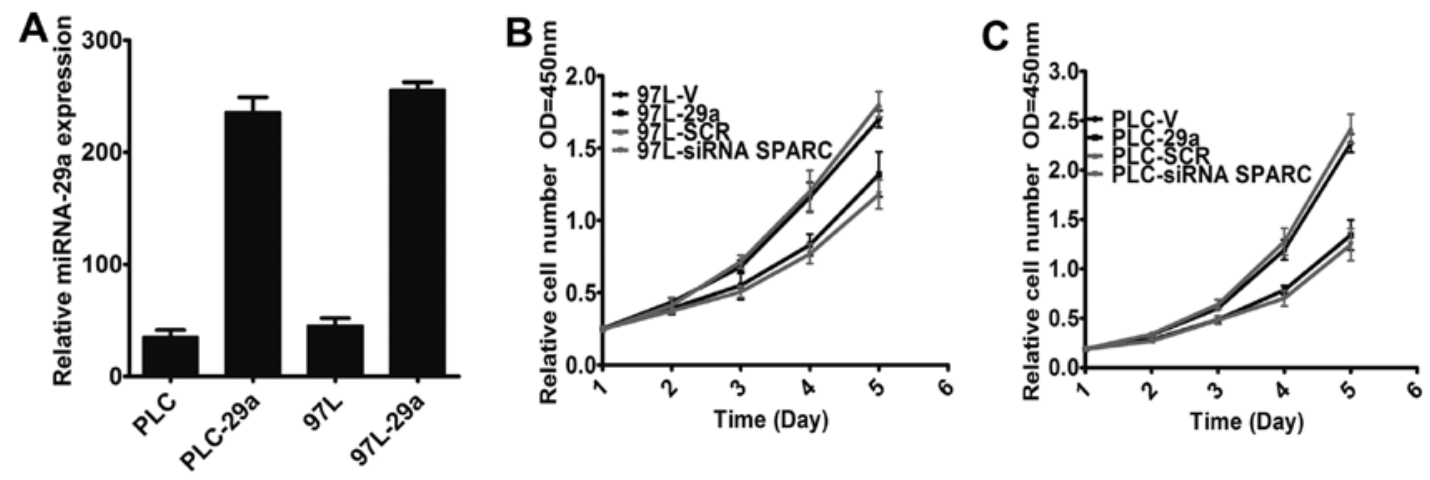

Figure 2. Overexpression of miR-29a suppresses HCC proliferation. (A) The expression of miR-29a was measured by TaqMan RT-PCR. After the cells were infected with a lentivirus containing miR-29a, RNA was extracted and assayed by qRT-PCR. Cell proliferation of the (B) MHCC-97L and (C) PLC cell lines was determined after infection with an miR-29a-expressing lentivirus or control lentivirus using WST-1 assays. These cells were assayed every day for 5 days. Statistical analysis was performed with Student's t-test. ${ }^{*} \mathrm{P}<0.05$

Statistical analyses. Data are expressed as the mean \pm standard error of the mean from at least three independent experiments. Kaplan-Meier estimate of the survival rate was contributed by SPSS software. All statistical tests were two-sided, and a $\mathrm{P}$-value $<0.05$ was considered to indicate a statistically significant result.

\section{Results}

miR-29a is downregulated in HCC. In a previous study, we observed differentially expressed miRNAs in metastatic versus non-metastatic liver tissues from patients with HCC (12). Here, we found that miR-29a was downregulated in HCC tissues from 110 patients compared to corresponding adjacent nontumor liver tissues using a specific TaqMan probe RT-PCR assay (Fig. 1A). There was a significant difference in miR-29a expression levels between HCC and the adjacent benign tissues $(\mathrm{P}<0.01)$. Next, we further analyzed the expression level of miR-29a in 4 human HCC cell lines compared to the LO2 normal immortalized liver cell line and found that miR-29a was expressed at significantly lower levels in HCC cells, especially in PLC and MHCC-97L, when compared with the LO2 cells (Fig. 1B). The SPARC mRNA levels were significantly higher in the HCC tissues than that in the adjacent non-tumor liver tissues $(\mathrm{P}<0.01)$ (Fig. 1C).

Overexpression of miR-29a suppresses HCC cell growth. To gain insight into the biological role of miR-29a in the regulation of cell growth in HCC, we constructed a lentivirus vector encoding the miR-29a precursor and established two stably infected cell lines, denoted PLC-29a and 97L-29a, after lentivirus infection. The expression level of miR-29a was then detected by TaqMan PCR after overexpression of miR-29a. The data showed that the expression levels of miR-29a in PLC-29a and 97L-29a were markedly increased, while miR-29a expression was not affected in the empty vectorinfected cells (Fig. 2A). To explore the role of miR-29a in HCC cell growth, we analyzed the regulatory effect of miR-29a on cell growth and found that both PLC-29a and 97L-29a cells grew at significantly lower rates than matched cells infected with the empty vector (Fig. 2B and C) $(\mathrm{P}<0.01)$.

SPARC is a direct target of miR-29a. To explore the possible molecular mechanism by which miR-29a suppresses cell growth, we analyzed SPARC mRNA, which is a predicted target of miR-29a. First, we measured SPARC protein levels 


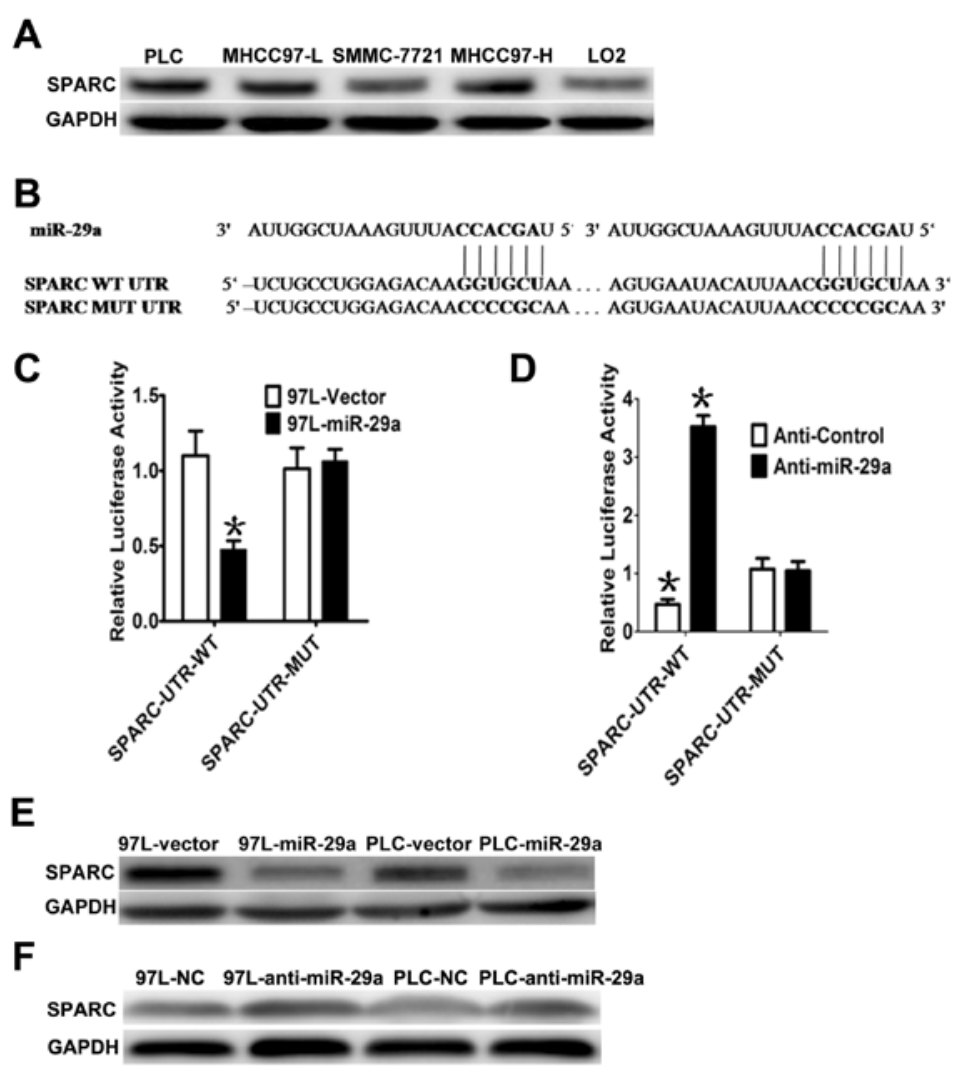

Figure 3. miR-29 downregulates SPARC expression by directly targeting its 3'UTR in HCC cells. (A) The protein levels of SPARC were determined by western blotting in the indicated cell lines. (B) Putative miR-29a binding site in the SPARC-3'UTR. Mutations were generated in the SPARC mRNA 3'UTR sequence in the complementary site for the seed region of miR-29a, the proximal UTR mutation bases (104-110), and the distal UTR mutation bases (137-143), as indicated. (C) Analysis of luciferase activity; the Renilla luciferase activity was normalized to firefly luciferase activity. MHCC-97L cells infected with vector control or miR-29a were transfected with a luciferase reporter containing the wild-type or mutant 3'UTR of the SPARC mRNA. (D) Luciferase activities were assayed in the MHCC-97L cells stably expressing miR-29a that were co-transfected with a luciferase reporter containing the wild-type or mutant 3'UTR and anti-miR-29a or anti-miR-NC (control). (E) SPARC protein levels were measured by western blotting in MHCC-97L and PLC cells infected with miR-29a or an empty vector construct. (F) SPARC protein levels were measured in MHCC-97L and PLC cells stably expressing miR-29a but treated with anti-miR-NC (control) or anti-miR-29a for $96 \mathrm{~h} .{ }^{*} \mathrm{P}<0.05$

in HCC cells and found that SPARC was highly expressed in the 4 HCC cell lines, but lowly expressed in normal liver LO2 cells (Fig. 3A). Interestingly, miR-29a and SPARC expression levels were inversely correlated in the $\mathrm{LO} 2$ cells and the $4 \mathrm{HCC}$ cell lines. To determine whether SPARC is a direct target of miR-29a, a Dual-Luciferase reporter system was employed. Using miRNA target prediction, we found that the 3'UTR of SPARC contains two miR-29a-binding sites (Fig. 3B). To assess miRNA binding to the 3'UTR, we constructed luciferase reporters with the SPARC-3'UTR. Co-transfection of the reporter containing the SPARC-3'UTR-WT construct with miR-29a significantly suppressed the Renilla luciferase activity in 97L-29a cells compared to the control group (empty vector), while the mutant SPARC-3'UTR reporter (i.e., mutated in the seed sequence binding region) displayed no response to high levels of miR-29a in 97L-29a cells (Fig. 3B and C). Similar results were also observed in the PLC-29a cells. Subsequently, we co-transfected anti-miR-29a and SPARC-3'UTR-WT or SPARC-3'UTR-MUT in miR-29a-overexpressing MHCC-97L (97L-29a) cells and observed that the anti-miR-29a inhibitor rescued the luciferase activities of the reporter containing the wild-type SPARC-3'UTR (Fig. 3D), but the mutant did not. In accordance with these results, we observed a clear decrease in endogenous SPARC protein in PLC and MHCC-97L cells with miR-29a overexpression, whereas no obvious changes were detected in the PLC and MHCC-97L cells infected with the vector (Fig. 3E). In addition, suppression of miR-29a by antisense miR-29a led to higher expression of SPARC (Fig. 3F). These results suggest that miR-29a downregulates SPARC expression, by direct targeting of its 3'UTR.

miR-29a inhibits cell growth via the SPARC-AKT pathway. Since SPARC is a positive regulator of AKT and mediates cell survival (5), and miR-29a can directly target SPARC mRNA and suppress HCC growth, the effectors downstream of SPARC required further investigation. As the downstream effector, AKT activation is sufficient to promote cell proliferation and survival. We hence examined the regulatory effect on and changes in P-AKT levels in PLC and MHCC-97L cells by miR-29a overexpression. The expression of P-AKT (S473), P-ERK (T202 and Y204) and P-mTOR (S2448) was significantly reduced in the PLC-29a and 97L-29a cells (Fig. 4A). These observations suggest that the AKT/mTOR pathway was inhibited in miR-29a-overexpressing HCC cells. To determine the role of SPARC in the AKT/mTOR pathway further, SPARC was knocked down to examine the importance of endogenous 

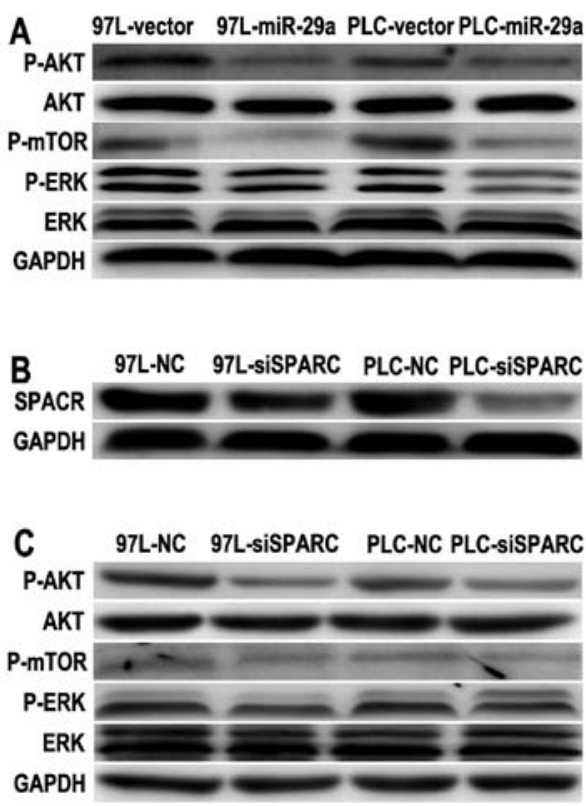

Figure 4. SPARC is involved in miR-29a-suppressed proliferation. (A) The endogenous protein levels of P-AKT (S473), P-mTOR (S2448) and P-ERK (T202,Y204) were determined by western blotting after infection with miR-29a or empty vector. GAPDH served as an internal control. The results are representative of at least three independent experiments using MHCC-97L and PLC cells stably expressing miR-29a or the vector control. (B) Endogenous SPARC expression was assayed by western blotting in cells expressing siRNA directed against SPARC. MHCC-97L and PLC cells were transfected with siSPARC or NC (control) for $48 \mathrm{~h}$ and then analyzed by western blotting (GAPDH, internal control). (C) The expression levels of P-AKT (S473), P-mTOR (S2448) and P-ERK (T202,Y204) in MHCC-97L and PLC cells were measured by western blotting after transfection with siRNA against SPARC or with NC (control).

SPARC expression. SPARC expression was greatly reduced after SPARC siRNA transfection in PLC and MHCC-97L cells (Fig. 4B). Silencing of SPARC significantly reduced both its protein levels and the cell growth rate (Fig. 2B), which was similar to the phenotype induced by miR-29a overexpression. Thus, we investigated the AKT/mTOR pathway, and found it was also suppressed after SPARC silencing in both MHCC-97L and PLC cells (Fig. 4C). These results suggest that SPARC silencing resulted in a similar effect on the AKT/ mTOR pathway as miR-29a overexpression.

miR-29a expression is associated with patient survival. All of the 110 patients enrolled in this study were divided into two groups according to the level of miR-29a expression. The 60 patients with miR-29a expression less than the median value were considered to have low expression of miR-29a, while 50 patients with miR-29a expression higher than the median level measured by PCR were considered to have high expression of miR-29a. After analysis with Kaplan-Meier method, we found that the patients with relatively high expression of miR-29a survived at a significantly higher rate than those with low expression of miR-29a in a 6-year survival surveillance (Fig. 5). Patients with higher miR-29a expression than the median level had a more favorable survival rate as compared with those with less than the median. Between the metastasis and no metastasis groups the difference in miR-29a expression was not significant $(\mathrm{P}=0.068)$.

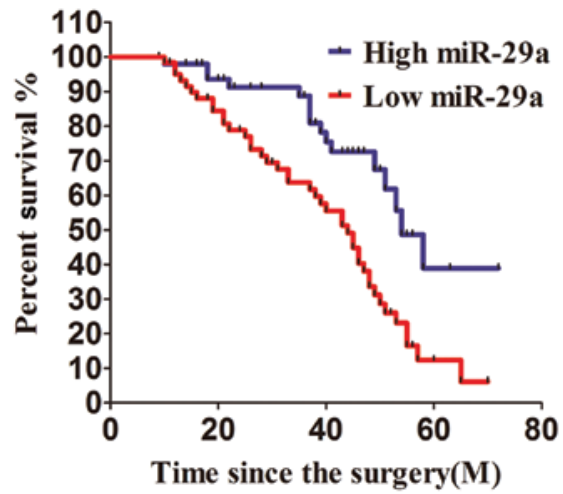

Figure 5. Kaplan-Meier survival analysis of the patients with HCC. A total of 110 patients were divided into 2 groups: the high expression group and the low expression (below the median level of miR-29a expression) group. The difference between the two groups reached statistical significance $(\mathrm{P}<0.01)$. M, month.

\section{Discussion}

Evidence shows that miRNAs have important roles in mRNA stability, directly contribute to mRNA degradation and gene expression (23). In this study, we found that miR-29a expression was reduced in HCC tissues compared to normal tissues and reduced in $4 \mathrm{HCC}$ cell lines compared to a normal liver cell line. Hence, we further investigated the molecular mechanisms involved in miR-29a action. To this end, we constructed an expression vector and established two cell models stably overexpressing miR-29a, which were used for cell growth regulation. Overexpression of miR-29a significantly inhibited the proliferation rate of both MHCC-97L and PLC cells. These data agree with previous reports indicating that miR-29 sensitizes HCC cells to apoptosis triggered by various stimuli (14).

There are lines of evidence indicating that miR-29a plays an important role in liver fibrosis and in sensitizing HCC cells to apoptosis $(14,24)$, but some reports also indicate that miR-29a promotes HCC cell invasion and metastasis by downregulating the tumor suppressor PTEN (25). These discrepancies may be due to differences among the samples, such as the sample source, diverse pathogenic attributes and miscellaneous pathological characteristics. Because one miRNA can target dozens of mRNAs that impact several molecules involved in various signaling pathways, the dominant influence of an miRNA on the regulation of cellular functions may depend on the relative importance of the targets that are involved in the signaling pathways (9). Indeed, the roles of an miRNA may oscillate between repression and activation in coordination with other regulators that catalyze the phosphorylation of the targets, and the roles of the miRNA also depend on the relative importance of the targeted molecule in the regulatory pathway (10).

SPARC is an extracellular glycoprotein which is involved in promoting cell motility and invasion in several carcinoma cell types, including breast, prostate (6), melanoma (4) and glioblastomas (5). SPARC overexpression in this study was observed in the HCC cell lines compared to the immortalized normal liver cell LO2, or in HCC tissues from 110 patients compared to the adjacent tissues. To elucidate the targets of miR-29a in HCC, we analyzed SPARC as a potential target of miR-29a. Using a luciferase reporter assay, we found that 
the 3'UTR from SPARC mRNA was significantly targeted by miR-29a. Mutation of the miR-29a binding sequence in the SPARC-3'UTR abrogated the effects of miR-29a. Use of an miR-29a antisense oligonucleotide rescued the activities of the luciferase reporter. Furthermore, in both HCC samples and HCC cell lines, miR-29a expression inversely correlated with SPARC expression. All of these data indicated that SPARC mRNA was targeted by miR-29a in HCC cells. Interestingly, the effect of SPARC siRNA on HCC cell growth was similar to miR-29a overexpression in both PLC and MHCC-97L cells. Thus, we believe that SPARC is the target by which miR-29a inhibits HCC cell proliferation.

SPARC can mediate cell survival through the activation of AKT (5). Activated AKT is sufficient to promote cell proliferation and survival since activated AKT phosphorylates MDM2 and inactivates p53. The role of the PI3K-Akt cascade in the regulation of proliferation, survival, and differentiation of many different cell types has been well established. Cumulative evidence indicates that abnormal activation of the PI3K/Akt/mTOR signaling pathway, which can promote cell growth, frequently occurs in HCC. In our study, we noted that overexpression of miR-29a reduced the level of AKT phosphorylation, but anti-miR-29a enhanced the level of P-AKT. The expression of miR-29a appears to be an independent prognostic factor for overall survival since a higher expression level of miR-29a was associated with increased patient survival.

Taken together, our results demonstrate that overexpression of miR-29a significantly inhibits HCC cell proliferation in vitro by inhibiting SPARC, a direct and functional target of miR-29a, which markedly promotes HCC cell proliferation. These findings may facilitate the development of potential therapeutics against HCC.

\section{References}

1. Burak KW and Kneteman NM: An evidence-based multidisciplinary approach to the management of hepatocellular carcinoma (HCC): the Alberta HCC algorithm. Can J Gastroenterol 24: 643-650, 2010.

2. Li SF, Hawxby AM, Kanagala R, Wright $H$ and Sebastian A: Liver transplantation for hepatocellular carcinoma: indications, bridge therapy and adjuvant therapy. J Okla State Med Assoc 105: 12-16, 2012.

3. Bouzin $\mathrm{C}$ and Feron O: Targeting tumor stroma and exploiting mature tumor vasculature to improve anti-cancer drug delivery. Drug Resist Updat 10: 109-120, 2007.

4. Alonso SR, Tracey L, Ortiz P, et al: A high-throughput study in melanoma identifies epithelial-mesenchymal transition as a major determinant of metastasis. Cancer Res 67: 3450-3460, 2007.

5. Shi Q, Bao S, Maxwell JA, et al: Secreted protein acidic, rich in cysteine (SPARC), mediates cellular survival of gliomas through AKT activation. J Biol Chem 279: 52200-52209, 2004.

6. Thomas R, True LD, Bassuk JA, Lange PH and Vessella RL: Differential expression of osteonectin/SPARC during human prostate cancer progression. Clin Cancer Res 6: 1140-1149, 2000.
7. Chan SK, Griffith OL, Tai IT and Jones SJ: Meta-analysis of colorectal cancer gene expression profiling studies identifies consistently reported candidate biomarkers. Cancer Epidemiol Biomarkers Prev 17: 543-552, 2008.

8. Lau CP, Poon RT, Cheung ST, Yu WC and Fan ST: SPARC and Hevin expression correlate with tumour angiogenesis in hepatocellular carcinoma. J Pathol 210: 459-468, 2006.

9. Jia CY, Li HH, Zhu XC, et al: MiR-223 suppresses cell proliferation by targeting IGF-1R. PLoS One 6: e27008, 2011.

10. Wu LH, Li HH, Jia CY, et al: MicroRNA-223 regulates FOXO1 expression and cell proliferation. FEBS Lett 586: 1038-1043, 2012.

11. Meng F, Henson R, Wehbe-Janek H, Ghoshal K, Jacob ST, Patel T: MicroRNA-21 regulates expression of the PTEN tumor suppressor gene in human hepatocellular cancer. Gastroenterology 133: 647-658, 2007.

12. Budhu A, Jia HL, Forgues M, et al: Identification of metastasisrelated microRNAs in hepatocellular carcinoma. Hepatology 47: 897-907, 2008

13. Hou J, Lin L, Zhou W, et al: Identification of miRNomes in human liver and hepatocellular carcinoma reveals miR-199a/b$3 \mathrm{p}$ as therapeutic target for hepatocellular carcinoma. Cancer Cell 19: 232-243, 2011.

14. Xiong Y, Fang JH, Yun JP, et al: Effects of microRNA-29 on apoptosis, tumorigenicity, and prognosis of hepatocellular carcinoma. Hepatology 51: 836-845, 2010.

15. Li R, Qian N, Tao K, You N, Wang X and Dou K: MicroRNAs involved in neoplastic transformation of liver cancer stem cells. J Exp Clin Cancer Res 29: 169, 2010.

16. Wang B,Komers R, Carew R, et al: Suppression of microRNA-29 expression by TGF-beta1 promotes collagen expression and renal fibrosis. J Am Soc Nephrol 23: 252-265, 2012.

17. Zhang P, Huang A, Ferruzzi J, et al: Inhibition of microRNA-29 enhances elastin levels in cells haploinsufficient for elastin and in bioengineered vessels - brief report. Arterioscler Thromb Vasc Biol 32: 756-759, 2012.

18. Papadopoulou AS, Dooley J, Linterman MA, et al: The thymic epithelial microRNA network elevates the threshold for infectionassociated thymic involution via miR-29a mediated suppression of the IFN-alpha receptor. Nat Immunol 13: 181-187, 2012.

19. Teichler S, Illmer T, Roemhild J, Ovcharenko D, Stiewe T and Neubauer A: MicroRNA29a regulates the expression of the nuclear oncogene Ski. Blood 118: 1899-1902, 2011.

20. Pandey AK, Verma G, Vig S, Srivastava S, Srivastava AK and Datta M: miR-29a levels are elevated in the $\mathrm{db} / \mathrm{db}$ mice liver and its overexpression leads to attenuation of insulin action on PEPCK gene expression in HepG2 cells. Mol Cell Endocrinol 332: 125-133, 2011.

21. Desjobert C, Renalier MH, Bergalet J, et al: MiR-29a downregulation in ALK-positive anaplastic large cell lymphomas contributes to apoptosis blockade through MCL-1 overexpression. Blood 117: 6627-6637, 2011.

22. Mott JL, Kurita S, Cazanave SC, Bronk SF, Werneburg NW and Fernandez-Zapico ME: Transcriptional suppression of mir-29b-1/ mir-29a promoter by c-Myc, hedgehog, and NF-kappaB. J Cell Biochem 110: 1155-1164, 2010.

23. Huang $S$ and He $X$ : The role of microRNAs in liver cancer progression. Br J Cancer 104: 235-240, 2011.

24. Roderburg C, Urban GW, Bettermann K, et al: Micro-RNA profiling reveals a role for miR-29 in human and murine liver fibrosis. Hepatology 53: 209-218, 2011.

25. Kong G,Zhang J, Zhang S, Shan C, Ye L and Zhang X: Upregulated microRNA-29a by hepatitis B virus X protein enhances hepatoma cell migration by targeting PTEN in cell culture model. PLoS One 6: e19518, 2011. 